\title{
Viel Lärm um Nichts?
}

\section{Der Einfluss der Elterngeldreform auf die Inanspruchnahme von Elternzeit durch Väter*}

\section{Von Kerstin Pull und Ann-Cathrin Vogt}

Zusammenfassung: Seit der Einführung des Elterngeldes zum 01. Januar 2007 ist der Anteil der Väter in Elternzeit über das gesamte Jahr 2007 hinweg zwar deutlich angestiegen; dennoch stellen Väter in Elternzeit nach wie vor die Minderheit. An diesem Punkt setzt der vorliegende Beitrag an und untersucht, wie die Elterngeldreform 2007 die Entscheidung eines berufstätigen Vaters für oder wider die Inanspruchnahme von Elternzeit beeinflusst hat. Als Ergebnis der empirischen Analyse, in deren Verlauf 1.290 Online-Fragebögen berufstätiger Väter ausgewertet wurden, zeigt sich, dass die Elterngeldreform die Wahrscheinlichkeit der väterlichen Inanspruchnahme von Elternzeit zwar signifikant erhöht hat, dass Väter seit Inkrafttreten der Reform aber im Durchschnitt kürzer in Elternzeit gehen, da sie im Wesentlichen die so genannten Vätermonate in Anspruch nehmen. Dies gilt auch nach Kontrolle einer Vielzahl potenzieller Determinanten der väterlichen Inanspruchnahme von Elternzeit aus dem beruflichen, familiären und gesellschaftlich-sozialen Umfeld sowie nach Kontrolle von Persönlichkeitsfaktoren. Was die übrigen Einflussfaktoren der väterlichen Inanspruchnahme von Elternzeit anbelangt, so gibt unsere Analyse erste tentative Hinweise darauf, dass die Elterngeldreform insofern zu einer Veränderung des väterlichen Entscheidungskalküls geführt hat, als dass auf der einen Seite vormals wirksame Determinanten des Kalküls in ihrer Wirkung abgeschwächt wurden (u.a. Einkommen des Vaters im Vergleich zur Mutter, Extraversion des Vaters) und auf der anderen Seite ursprünglich weniger bedeutsame Determinanten in ihrer Wirksamkeit gestärkt wurden (u.a. zeitliche Belastung des Vaters im Beruf, Verträglichkeit des Vaters).

\section{Einführung}

Das Thema Elternzeit, insbesondere was die Beteiligung der Väter betrifft, erfährt derzeit eine große Aufmerksamkeit in den Medien: Wurde bislang die Vereinbarkeit von Beruf und Familie im Wesentlichen als Problem der Frauen angesehen, so wird heute zunehmend der väterliche Beitrag an der Kinderbetreuung diskutiert. Dabei ist zwar seit der Einführung des Elterngeldes zum 01. Januar 2007 der Anteil der Väter in Elternzeit in 2007 von rund vier auf zehn Prozent angestiegen (BMFSFJ 2008 a; BMFSFJ 2004: 20), dennoch stellen Väter in Elternzeit nach wie vor die Minderheit. An diesem Punkt setzt der vorliegende Beitrag an und untersucht, wie die Elterngeldreform 2007 die Entscheidung eines berufstätigen Vaters für oder wider die Inanspruchnahme von Elternzeit beeinflusst hat. Dabei wird zum einen der direkte Einfluss der Elterngeldreform auf die Inanspruchnahme von Elternzeit durch Väter untersucht, und zum anderen wird analysiert, in welcher Weise die Elterngeldreform das Entscheidungskalkül berufstätiger Väter für oder wider Elternzeit in dem Sinne beeinflusst haben mag, dass vormals wirksame Determinanten des Kalküls in ihrer Wirkung abgeschwächt bzw. ursprünglich weniger bedeutsame Determinanten in ihrer Wirksamkeit gestärkt wurden.

\section{Stand der Literatur zu den Determinanten der familiären Arbeitsteilung}

Die Familie ist ein klassisches Untersuchungsthema der Sozialwissenschaften. Insbesondere das letzte Vierteljahrhundert ist durch ein immer größer werdendes Interesse an Mutter- und

* Wir danken einem anonymen Gutachter für seine hilfreichen Hinweise sowie der Hanns-Seidel-Stiftung für die Förderung des Projektes über ein Begabtenstipendium aus Mitteln des Bundesministeriums für Bildung und Forschung (BMBF). 
Vaterschaft gekennzeichnet (Palkovitz / Marks 2002: 144). Die Vater-Kind-Beziehung wurde dabei erst vergleichsweise spät Gegenstand wissenschaftlichen Interesses (Gornick / Meyers 2008). Während dem Vater bis Mitte der 70er Jahre nahezu ausschließlich die Rolle des Familienernährers zugewiesen wurde (Griswold 1993: 13), fand zu Beginn der 80er Jahre verstärkt die Eignung des Mannes, seinen familiären Fürsorgepflichten nachzukommen, Beachtung (Metz-Göckel 1988, Peinelt-Jordan 1996). So galt dann auch das besondere Interesse Fthenakis (1985) den so genannten „Neuen Vätern“, die aus den traditionellen Vaterrollen ausbrechen und sich den geänderten Ansprüchen an ihre Vaterpflichten stellen (vgl. auch Coltrane 1996; Pleck 1997; Sayer / Bianchi / Robinson 2004). Der Vater wurde als Gegenstand der Familienforschung quasi ,neu entdeckt“. Seit den 90er Jahren befassen sich etliche Arbeiten mit der Vaterrolle in der Familie und der familiären Arbeitsteilung (z.B. Oberndorfer 1993; Boeykens 1993; Blankenhorn 1995; Benard / Schlaffer 1991); dieser Trend setzt sich bis heute fort.

Zum gegenwärtigen Zeitpunkt liegt eine Vielzahl an Studien vor, die sich mit den Determinanten der familiären Arbeitsteilung befasst. Theoretisch basieren die vorliegenden Arbeiten auf unterschiedlichen Theoriesträngen. Besonders bedeutsam sind dabei der Geschlechterrollen-Ansatz, der Zeitbudget-Ansatz sowie die ökonomische Theorie der Familie (Hill / Kopp 1995; Künzler 1994; Höpflinger / Charles 1990). Neben dem institutionellen Kontext wurden in den vorliegenden Arbeiten (1) das berufliche Umfeld (Einkommen, Bildungsabschluss, zeitliche Belastung etc.), (2) das familiäre Umfeld (Familienorientierung, zeitlicher Aufwand für Hausarbeit, Erwerbstätigkeit der Mutter etc.), (3) das gesellschaftlich-soziale Umfeld (Modernität des Geschlechterrollenverständnisses, soziale Akzeptanz etc.) und (4) persönliche Faktoren (z.B. Alter, Erfahrungen, Wünsche des Vaters) als Determinanten der familiären Arbeitsteilung identifiziert. In unserem Beitrag berücksichtigen wir potenzielle Determinanten aus den unterschiedlichen Bereichen:

(1) Was das berufliche Umfeld anbelangt, so untersuchen wir erstens das Einkommen des Vaters im Vergleich zu dem der Mutter (z.B. Vaskovics / Rost 1999; Vaskovics / Rupp 1995; Bianchi et al. 2000; Sundström / Duvander 2002; Ondrich / Spieß / Yang 1996; Künzler 1994), zweitens ob Vater oder Mutter über einen höheren Bildungsabschluss verfügen (z.B. Merz 2004; Bianchi et al. 2000; Lappegard 2008; Yeung et al. 2001; Sundström / Duvander 2002; Kreyenfeld / Geisler 2009), drittens ob zum Zeitpunkt der Entscheidung für oder wider Elternzeit konkrete Karriereschritte bei Vater und / oder Mutter anstanden (z.B. Kirchmeyer 2002; BMFSFJ 2004, Behnke / Meuser 2003), viertens die zeitliche Belastung des Vaters im Beruf und schließlich fünftens die Arbeitgeberorientierung des Vaters (z.B. Pylkkänen / Smith 2003; Künzler 1994; Hofferth 2003; Yeung et al. 2001).

(2) Was das familiäre Umfeld anbelangt, so berücksichtigen wir sowohl eine Variable zur Familienorientierung des Vaters als auch den zeitlichen Aufwand für Hausarbeit von Vater und Mutter zum Zeitpunkt der Entscheidung über eine Inanspruchnahme von Elternzeit durch den Vater (z.B. Coverman 1985, Engelhardt / Jan 2004).

(3) Was das gesellschaftlich-soziale Umfeld anbelangt, so fragen wir nach der Modernität des Geschlechterrollenverständnis des Vaters (z.B. Bulanda 2004; Döge / Volz 2002; Hofferth 2003; Künzler 1994) sowie danach, in welchem Umfang der Vater bei Inanspruchnahme von Elternzeit mit Karrierehindernissen rechnet (als Indikator für die soziale Akzeptanz der Inanspruchnahme von Elternzeit; z.B. Oberndorfer / Rost 2002; Peinelt-Jordan 1996; Vaskovics / Rost 1999; Spieß / Wrohlich 2008).

(4) Bezüglich der persönlichen Faktoren fragen wir - vor dem Hintergrund der zunehmenden Beachtung, welche der Berücksichtung von Persönlichkeitsfaktoren bei der Analyse von Entscheidungsprozessen zukommt - nach den Persönlichkeitsmerkmalen des Vaters. $\mathrm{Zu}$ deren Erfassung nutzen wir den in der Persönlichkeitspsychologie weit verbreiteten Big-Five-Ansatz (siehe etwa Schumann 2005), welcher die fünf bipolaren Di- 
mensionen Extraversion, Gewissenhaftigkeit, Verträglichkeit, Neurotizismus und Offenheit für Erfahrung umfasst.

Mit dieser Auswahl an Faktoren decken wir weite Teile der in der Literatur herausgearbeiteten Determinanten der familiären Arbeitsteilung ab und erweitern diese zugleich um die Berücksichtigung der Persönlichkeitsmerkmale des Vaters als mutmaßlich weitere bedeutsame Determinanten der väterlichen Entscheidung für oder wider die Inanspruchnahme von Elternzeit.

\section{Die Elterngeldreform 2007}

Mit der Einführung des Elterngeldes zum 1.1.2007 wurden die gesetzlichen Regelungen für die Inanspruchnahme von Elternzeit grundlegend reformiert: Das sog. Bundeselterngeldgesetz trat zum 1.1.2007 in Kraft und an die Stelle des Bundeserziehungsgeldgesetzes. Es gilt für alle ab dem 1.1.2007 geborenen Kinder (BMFSFJ 2007: 7ff). Das Erziehungsgeld, welches vor dem 1.1.2007 galt, wurde in der Regel für längstens zwei Jahre gewährt und betrug maximal 300 Euro pro Monat. Der Höchstbetrag wurde dann bezahlt, wenn das Haushaltseinkommen unter 30.000 Euro jährlich lag. Vom siebten Lebensmonat des Kindes an lag die Einkommensgrenze niedriger: Ab einem jährlichen Haushaltseinkommen von 16.500 Euro wurde das Erziehungsgeld für jeden zusätzlichen Euro Einkommen im Monat um 63 Cent gekürzt. (BMFSFJ 2007: 7ff)

Anders als das Erziehungsgeld ist das Elterngeld eine Einkommensersatzleistung, d.h. demjenigen Elternteil, der seine Erwerbstätigkeit für die Kinderbetreuung unterbricht, wird beim Elterngeld der entstandene Einkommensausfall in Höhe von 67\% des Nettoeinkommens der letzten zwölf Kalendermonate vor der Geburt des Kindes ersetzt. Reduziert ein Elternteil seine Erwerbstätigkeit zur Inanspruchnahme von Elternzeit, wird nur das Einkommen ersetzt, das durch die Einschränkung der Erwerbstätigkeit wegfällt. Das monatliche Elterngeld beträgt maximal 1.800 Euro und mindestens 300 Euro im Monat. Der Mindestsatz gewährleistet, dass auch jene Elternteile eine Leistung erhalten, die vor der Geburt des Kindes nicht erwerbstätig waren. Für Elterngeldbezieher, die vor der Geburt des Kindes ein monatliches Nettoeinkommen von unter 1.000 Euro hatten, wird die Ersatzrate schrittweise auf bis zu 100\% angehoben. (BMFSFJ 2007: 3; 7ff; 17; BMFSFJ 2008 b: 55)

Im Unterschied zum Erziehungsgeld wird das Elterngeld für maximal 14 Monate gewährt, wobei ein Elternteil maximal 12 Monate Elterngeld beziehen kann. Die zwei verbleibenden Monate, sog. Partnermonate, sind nur vom anderen Elternteil nutzbar. Da es in der Regel die Mutter ist, die die Elternzeit beansprucht, sind diese Partnermonate auch unter dem Begriff „Vätermonate“ bekannt geworden. Denn die zwei Monate, für die nur dann Elterngeld ausgezahlt wird, wenn sowohl Mutter als auch Vater jeweils mindestens zwei Monate vom Elterngeld Gebrauch machen, sollen insbesondere Väter ermutigen, Elternzeit zu nutzen.

Es ist davon auszugehen, dass die Elterngeldreform die ökonomische Anreizsituation - insbesondere was die Elternzeit-Inanspruchnahme gut verdienender Väter anbelangt - seit dem 1.1.2007 maßgeblich verändert hat. Dabei ist zu vermuten, dass die ökonomischen Anreize einer Inanspruchnahme von Elternzeit durch Väter seit dem 1.1.2007, d.h. seit Bestehen des Elterngelds in Höhe von $67 \%$ des Nettoeinkommens, angestiegen sind. Gründe für diese Vermutung sind zweierlei:

Erstens wird der Einkommenshöhe des Vaters bei der Berechnung des Elterngelds seit dem 1.1.2007 erstmals eine Bedeutung beigemessen; zwischen dem Einkommen des Anspruchsberechtigten und der Höhe des Elterngelds besteht bei der Entscheidung über Elternzeit seit dem 1.1.2007 ein positiver Zusammenhang. Ein hohes Einkommen vor Antritt der Elternzeit zahlt sich also bei einer Erwerbsunterbrechung für ein nach dem 1.1.2007 geborenes Kind durch ein höheres Elterngeld aus (siehe auch Spieß 2006: 69). Das hieße, für gut verdienende 
Eltern würde das Elterngeld höher ausfallen als die finanzielle Leistung, die sie unter der Erziehungsgeldregelung hätten beziehen können. Unter der Annahme, dass Väter in der Regel mehr verdienen als ihre Partnerinnen und ihnen daher im Falle einer Inanspruchnahme von Elternzeit ein höheres Elterngeld gewährt werden würde als ihren Partnerinnen, lässt sich vermuten, dass der Anteil der Väter, die nach 2007 Elternzeit beanspruchen, steigt. Zweitens sind an dieser Stelle aber auch die mit der Elterngeldreform eingeführten sog. Vätermonate nicht zu vernachlässigen, von denen angenommen werden kann, dass sie die ökonomische Anreizsituation, Elternzeit zu beanspruchen, seit 2007 zusätzlich veränderten und den Anteil an männlichen Elterngeld-Nutzern erhöhten. Möchten Eltern die maximale Elterngeldbezugsdauer von 14 Monaten voll ausschöpfen, müssen beide Elternteile für jeweils mindestens zwei Monate Elternzeit nutzen.

Angesichts der Tatsache, dass das Elterngeld insgesamt finanziell attraktiver geworden ist und die Einführung der Vätermonate einen weiteren finanziellen Anreiz für den Vater geschaffen hat, zumindest für zwei Monate Elternzeit in Anspruch zu nehmen, ist entsprechend zu erwarten, dass die Elterngeldreform die Entscheidung eines Vaters, Elternzeit in Anspruch zu nehmen, positiv beeinflusst. Dies führt zu folgender Hypothese:

Hypothese 1: Nach der Elterngeldreform ist die Wahrscheinlichkeit einer Inanspruchnahme von Elternzeit durch den Vater größer als vor der Reform.

Allerdings ist ebenfalls zu erwarten, dass Väter, die durch die Elterngeldreform zur Inanspruchnahme von Elternzeit motiviert werden, in vielen Fällen tatsächlich auch nur die zwei Vätermonate in Anspruch nehmen werden. Betrachtet man sodann die Gruppe der Elternzeit in Anspruch nehmenden Väter, so sinkt erwartbar die Wahrscheinlichkeit, dass ein Vater Elternzeit für eine Dauer von mehr als zwei Monaten in Anspruch nimmt. Entsprechend dürfte auch die durchschnittliche Dauer der Inanspruchnahme von Elternzeit durch Väter mit der Elterngeldreform zurück gegangen sein. Dies führt zu folgenden Hypothesen:

Hypothese 2: Nach der Elterngeldreform ist die Wahrscheinlichkeit, dass ein Vater Elternzeit mit einer Dauer von über zwei Monaten in Anspruch nimmt, geringer als vor der Reform.

Hypothese 3: Nach der Elterngeldreform ist die durchschnittliche Dauer der Inanspruchnahme von Elternzeit durch den Vater kürzer als vor der Reform.

Des Weiteren ist nicht auszuschließen, dass die Elterngeldreform auch die übrigen Determinanten der Entscheidung eines Vaters, Elterngeld in Anspruch zu nehmen, in ihrer Wirkung beeinflusst: So ist etwa denkbar, dass der Einfluss der Einkommensdifferenz zwischen Vater und Mutter mit Einführung der Vätermonate abgeschwächt wurde, da Elterngeld nunmehr einkommensabhängig gewährt wird. Auch könnte man sich vorstellen, dass der Zusammenhang zwischen Arbeitgeberorientierung und der Inanspruchnahme von Elternzeit nach der Elterngeldreform ein anderer ist als vorher. Gleiches gilt für die Persönlichkeitsfaktoren: Je mehr Väter Elternzeit in Anspruch nehmen, desto weniger „offen“ muss man als Vater möglicherweise sein, um den Schritt einer Inanspruchnahme von Elternzeit zu wagen. Inwiefern die Elterngeldreform neben direkten auch solche indirekten Wirkungen entfaltet, prüfen wir im Rahmen einer explorativen Vorher-Nachher-Analyse.

\section{Empirische Analyse}

\subsection{Datenbasis}

Basis der folgenden empirischen Analyse ist ein originärer Datensatz, welcher mithilfe einer 2008 durchgeführten Online-Befragung von Vätern in Deutschland mit mindestens einem seit dem 1.1.2001 geborenem Kind, die zum Zeitpunkt der Entscheidung über Elternzeit berufstätig waren und in einer Partnerschaft lebten. Insgesamt liegen 1.290 auswertbare Fragebögen vor, 
809 Väter gaben Auskunft darüber, ob sie die Entscheidung über eine Inanspruchnahme von Elternzeit vor oder nach 2007 trafen. Von den 809 Vätern entschieden 263 Väter vor und 546 nach dem 1.1.2007 über die Inanspruchnahme von Elternzeit. Während sich vor 2007 insgesamt 68 von 263 Vätern (25,9\%) für Elternzeit entschieden, waren es seit 2007 insgesamt 346 von 546 Vätern $(63,4 \%)$. Damit dürften Väter in Elternzeit in unserer Stichprobe deutlich überrepräsentiert sein.

\subsection{Operationalisierung der Variablen}

\section{Abhängige Variablen}

In unseren Schätzungen verwenden wir drei verschiedene abhängige Variablen: Die sich auf die Überprüfung von Hypothese 1 beziehende abhängige Variable „Inanspruchnahme von Elternzeit: ja (1) - nein (0)“ ist eine 0 / 1-Variable, welche den Wert „1“ annimmt, wenn der betreffende Vater nach 2007 Elternzeiterfahrung gesammelt hat, und den Wert „0“, wenn dies nicht der Fall ist. Die abhängige Variable zur Überprüfung von Hypothese 2 lautet entsprechend „Dauer der Inanspruchnahme > 2 Monate: ja (1) - nein (0)“, und die abhängige Variable zur Überprüfung von Hypothese 3 lautet „Dauer der Inanspruchnahme (in Monaten)“. In den Schätzungen zu Hypothese 2 und 3 sind jeweils nur die Datensätze von Vätern enthalten, die sich für eine Inanspruchnahme von Elternzeit entschieden haben.

\section{Erklärende Variablen}

Zentrale erklärende Variable zur Prüfung der Hypothesen ist die Variable „,nach der Reform: ja (1) - nein (0)“. Hierzu wurde im Fragebogen abgefragt, ob der Vater während der zuletzt in Anspruch genommenen Elternzeit Elterngeld (für ein nach dem 1.1.2007 geborenes Kind) oder Erziehungsgeld (für ein vor dem 1.1.2007 geborenes Kind) in Anspruch nahm oder ob sofern keine Elternzeit gewählt wurde - solches beansprucht hätte werden können ( $1=$ Elterngeld/ $0=$ Erziehungsgeld).

Die weiteren erklärenden Variablen wurden folgendermaßen operationalisiert:

- Einkommen des Vaters im Vergleich zu dem der Mutter: Was das zum Zeitpunkt der Entscheidung über Elternzeit erzielte Einkommen der befragten Väter anbelangt, so wurde - im Interesse der Erzielung einer hohen Rücklaufquote - nicht die absolute Höhe der Einkommen abgefragt, sondern lediglich, ob das Bruttojahreseinkommen des Vaters zum Entscheidungszeitpunkt höher, gleich hoch oder niedriger als das der Partnerin war. Zudem wurde in einer Folgefrage die absolute Höhe der Differenz der Brutto-Jahreseinkommen beider Elternteile abgefragt. In die multivariate Analyse geht die Einkommensdifferenz, d.h. die letztgenannte Variable ein, wobei das Vorzeichen der Differenz aus der erstgenannten Frage abgeleitet wird: Verdiente der Vater mehr als seine Partnerin, wurde die Einkommensdifferenz mit , "+ kodiert, verdiente er weniger als seine Partnerin, mit „,-“. Gab ein Vater an, dass er ,etwa gleich viel“" wie seine Partnerin verdiente und war die angegebene Einkommensdifferenz $<5.000$ Euro, so wurde dies als quasi gleich hohes Einkommen mit „0“ kodiert. 20 Befragungen, nach denen zwar ein ,etwa gleich hohes“ Einkommen besteht, aber zusätzlich eine Einkommensdifferenz $\geq 5.000$ Euro angegeben wurde, wurden aus den Auswertungen ausgeschlossen.

- Bildungsabschluss des Vaters im Vergleich zu dem der Mutter: Bezüglich der zum Entscheidungszeitpunkt erreichten höchsten Bildungsabschlüsse der Elternteile wurde der mögliche Antwortraum in eine überschaubare Anzahl an Bildungsabschlüssen vorgegliedert, denen sich die Befragten zuordnen konnten. Zusätzlich wurden die befragten Väter aufgefordert, anzugeben, ob ihre Partnerinnen einen höheren, gleich hohen oder 
niedrigeren Bildungsabschluss als sie selbst erreicht haben. In die multivariate Analyse ging sodann die Frage ein, ob ein Vater über einen höheren Bildungsabschluss als die Partnerin verfügt. Es handelt sich um eine Dummy-Variable, die die Ausprägung 1 für „der Vater hat einen höheren Bildungsabschluss als die Partnerin“ und 0 für ,der Vater hat einen gleich hohen oder niedrigeren Bildungsabschluss" annimmt.

- Geplante Karriereschritte: Der Vater wurde befragt, ob er und/oder seine Partnerin zum Zeitpunkt der Entscheidung über Elternzeit (weitere) Karriereschritte innerhalb der nächsten fünf Jahre planten ( $1=\mathrm{ja} / 0=$ nein $)$.

- Zeitliche Belastung im Beruf: Die befragten Väter wurden gebeten, die von ihnen bzw. ihrer Partnerin zum Zeitpunkt der Entscheidung über Elternzeit im Durchschnitt geleisteten Wochenarbeitsstunden anzugeben.

- Arbeitgeberorientierung: Die Arbeitgeberorientierung wurde mit dem Item „Ich identifiziere mich mit meinem Arbeitgeber" erfasst. Der Befragte gab seinen Grad der Zustimmung zu der Aussage anhand einer 5er-Likert-Skala von 1 für „Ich stimme gar nicht

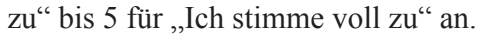

- Familienorientierung: Die Familienorientierung des Vaters wurde gemessen anhand des Zustimmungsgrades zum Item „Für die Familienharmonie mache ich alles“. Wieder gab der Befragte seine Zustimmung auf einer 5er-Likert-Skala von 1 für „Ich stimme gar nicht zu“ bis 5 für „Ich stimme voll zu“ an.

- Zeitlicher Aufwand für Hausarbeit: Die befragten Väter wurden gebeten, die von ihnen zum Zeitpunkt der Entscheidung über Elternzeit im Durchschnitt geleisteten wöchentlichen Stunden an Hausarbeit und die ihrer Partnerin anzugeben.

- Modernität des Geschlechterrollenverständnisses des Vaters: Das Geschlechterrollenverständnis des Vaters wurde anhand von drei auf einer 5er-Likert-Skala zu beurteilenden Aussagen (siehe Abbildung 1) gemessen, welche dann mithilfe einer exploratorischen Faktorenanalyse zu einem Faktor verdichtet wurden. Das MSA-Kriterium nach Kaiser / Meyer / Olkin ergibt den Wert von 0,616, was auf eine mittlere bis gute Eignung des gewählten Analyseverfahrens zur Zusammenfassung der Variablen hindeutet. Die Reliabilitätsanalyse über alle drei Items ergibt ein Cronbach-Alpha von 0,639 bei einer mittleren Inter-Item-Korrelation von 0,400. Es ist zu erkennen, dass die Items durch die Faktorenanalyse nicht an internem Zusammenhang verloren haben und sich die empirisch erhobenen rollentheoretischen Variablen für die Faktorenanalyse und der extrahierte Faktor zum Einsatz in die weiteren Analysen eignen.

Abbildung 1: Aussagen zur Modernität des Geschlechterrollenverständnisses des Vaters

1. Väter sollten sich heutzutage im familiären Bereich engagieren.

2. Kindererziehung ist nicht Frauensache.

3. Frauen sind für den finanziellen Unterhalt der Familie genauso verantwortlich wie Männer.

Quelle: Eigene Erstellung.

- Erwartete Karrierehindernisse: Was die Operationalisierung dieser Variable anbelangt, so wurden die an der Befragung partizipierenden Väter aufgefordert, die infolge der Inanspruchnahme von Elternzeit erwarteten Karrierehindernisse einzuschätzen. Hierzu wurden insgesamt sechs Aussagen formuliert (siehe Abbildung 2). Der befragte Vater gab seinen Zustimmungsgrad zu den Aussagen auf einer 5er-Likert-Skala von 1 für „Trifft gar nicht zu“ bis 5 für „Trifft sehr gut zu“ an. Zur Prüfung der Variablen wurden die sechs Aussagen dann mithilfe einer Faktorenanalyse zu einem Faktor verdichtet, der in die multivariate Analyse einfließt. Dass die sechs Items für faktorenanalytische Zwecke sehr gut geeignet sind, bestätigt das MSA-Kriterium, das für die Korrelationsmatrix 
einen Wert von 0,836 erbringt und damit die Korrelationsmatrix als sehr geeignet für die Faktorenanalyse deklariert. Als Extraktionsverfahren wurde hier und im Folgenden stets die Hauptkomponentenanalyse eingesetzt, deren Ziel in der möglichst umfassenden Reproduktion der Datenstruktur durch möglichst wenige Faktoren liegt. Die Reliabilitätsanalyse ergibt für die sechs Items ein Cronbach Alpha von 0,804.

Abbildung 2: Aussagen zu erwarteten Karrierehindernissen

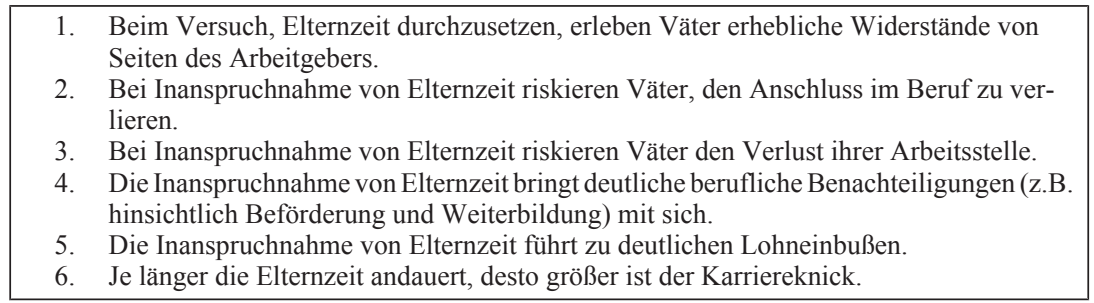

Quelle: Eigene Erstellung.

- Persönlichkeit des Vaters: Zur Erfassung der Persönlichkeitseigenschaften des Vaters wurde das Big Five-Inventar-Short version (BFI-S), welches von Gerlitz / Schupp (2005) für das Sozio-ökonomische Panel entwickelt wurde, eingesetzt. Die bewährte Fragebatterie umfasst 15 Items, je drei pro Faktor (siehe Abbildung 3). Die Aussagen zu den Persönlichkeitsmerkmalen wurden vom Vater auf einer 7er-Likert-Skala von 1 für „Trifft gar nicht zu“ bis 7 für „Trifft sehr gut zu“ beurteilt. Die jeweiligen drei zu einem Faktor gehörenden Items wurden dann mithilfe einer Faktorenanalyse verdichtet. Das MSA-Kriterium ergibt hier einen Wert von 0,702, was auf eine gute Eignung der 15 Big Five-Items für faktorenanalytische Zwecke schließen lässt. Im vorliegenden Fall ergibt die Reliabilitätsanalyse über alle drei Items des Faktors Extraversion ein Cronbach-Alpha von 0,744 bei einer mittleren Inter-Item-Korrelation von 0,506. Für die Faktoren Gewissenhaftigkeit, Verträglichkeit, Neurotizismus bzw. Offenheit ergeben sich Cronbach-Alpha-Werte von 0,603, 0,512, 0,649 bzw. 0,616 bei einer mittleren Inter-Item-Korrelation von 0,367, 0,283, 0,386 bzw. 0,366. Zwar liegen bis auf die Extraversion-Skala alle Skalen-Alpha-Werte unterhalb des konventionellen Wertes von 0,7 , allerdings zeigen die mittleren Inter-Item-Korrelationen, dass zwischen den Items aller Skalen ein relativ starker Zusammenhang besteht. Nach Gerlitz / Schupp (2005, S. $21 \mathrm{f}$ ) ist zu erkennen, dass die Items der Skalen durch die Faktorenanalyse nicht an internem Zusammenhang verloren haben. Die Cronbach-Alpha-Werte sind lediglich aufgrund der geringeren Itemanzahl niedriger (Gerlitz / Schupp 2005: 11). 
Abbildung 3: Big Five-Inventar-Short version zur Abfrage der Persönlichkeitseigenschaften des Vaters

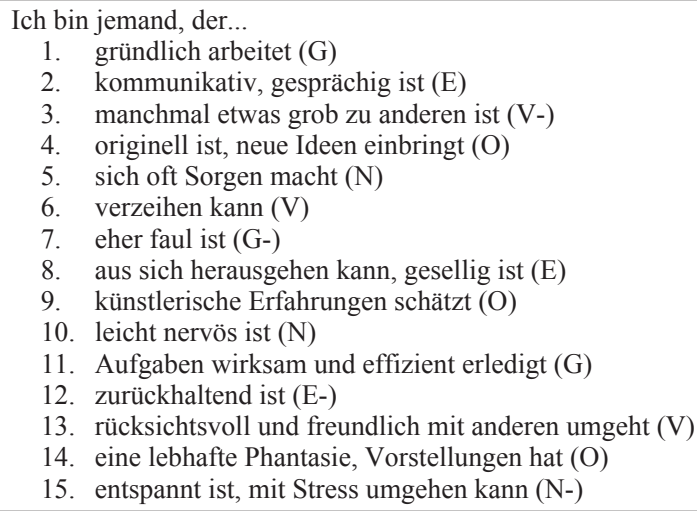

Legende: G: Gewissenhaftigkeit, E: Extraversion, V: Verträglichkeit, O: Offenheit für Erfahrung, $\mathrm{N}$ : Neurotizismus. Das Minuszeichen (-) zeigt die sprachlich negativ bzw. positiv gepolten Items an. Nach Abschluss der Datenerhebung und vor Durchführung der Faktorenanalyse wurden diese gepolten Item-Werte denen der anderen Items angepasst, d.h. "zurückgepolt".

Quelle: Gerlitz / Schupp (2005). 
Zusammenfassend liefert Tabelle 1 eine Übersicht über alle untersuchten Variablen sowie deren Mittelwerte und Mediane.

Tabelle 1: Übersicht über die verwendeten Variablen - Datendeskription

\begin{tabular}{|c|c|c|c|}
\hline Variable & Mittelwert & Median & Beschreibung/Wertebereich \\
\hline $\begin{array}{l}\text { Elterngeld nach } 2007 \text { oder Er- } \\
\text { ziehungsgeld vor } 2007\end{array}$ & 0,67 & 1,00 & $1=\operatorname{nach} 2007 ; 0=$ vor 2007 \\
\hline Einkommensdifferenz & $14.476 €$ & $10.000 €$ & $\begin{array}{l}\text { Metrische Variable (Textfeld im Zahlen- } \\
\text { format); verdiente der Vater mehr als sei- } \\
\text { ne Partnerin, wurde die angegebene Ein- } \\
\text { kommensdifferenz mit "+" kodiert, ver- } \\
\text { diente er weniger mit "-" }\end{array}$ \\
\hline $\begin{array}{l}\text { Bildungsabschluss } \\
\text { Vater_höher als bei P }\end{array}$ & 0,29 & 0,00 & $1=\mathrm{ja} ; 0=$ nein \\
\hline Karrierepläne & 0,55 & 1,00 & $1=\mathrm{ja} ; 0=$ nein \\
\hline Karrierepläne_P & 0,28 & 0,00 & $1=$ ja; $0=$ nein \\
\hline Wochenarbeitsstunden & 44,85 & 42,00 & $\begin{array}{l}\text { Metrische Variable (Textfeld im Zahlen- } \\
\text { format) }\end{array}$ \\
\hline Wochenarbeitsstunden_P & 36,20 & 40,00 & $\begin{array}{l}\text { Metrische Variable (Textfeld im Zahlen- } \\
\text { format) }\end{array}$ \\
\hline Arbeitgeberorientierung & 3,68 & 4,00 & $\begin{array}{l}\text { 5er-Likert-Skala von } 1 \text { für "Ich stimme } \\
\text { gar nicht zu" bis } 5 \text { "Ich stimme voll zu" }\end{array}$ \\
\hline Familienorientierung & 4,32 & 5,00 & $\begin{array}{l}\text { 5er-Likert-Skala von } 1 \text { für "Ich stimme } \\
\text { gar nicht zu" bis } 5 \text { "Ich stimme voll zu" }\end{array}$ \\
\hline $\begin{array}{l}\text { Geleistete Stunden } \\
\text { Hausarbeit }\end{array}$ & 9,09 & 8,00 & $\begin{array}{l}\text { Metrische Variable (Textfeld im Zahlen- } \\
\text { format) }\end{array}$ \\
\hline $\begin{array}{l}\text { Geleistete Stunden } \\
\text { Hausarbeit_P }\end{array}$ & 18,92 & 15,00 & $\begin{array}{l}\text { Metrische Variable (Textfeld im Zahlen- } \\
\text { format) }\end{array}$ \\
\hline $\begin{array}{l}\text { Modernität } \\
\text { Geschlechterrollenverständ- } \\
\text { nis }\end{array}$ & 4,32 & 5,00 & $\begin{array}{l}\text { Beurteilung von } 3 \text { Aussagen auf einer 5er- } \\
\text { Likert-Skala von } 1 \text { für "Ich stimme gar } \\
\text { nicht zu" bis } 5 \text { "Ich stimme voll zu", dann } \\
\text { Dimensionsreduzierung durch Faktoren- } \\
\text { analyse }\end{array}$ \\
\hline $\begin{array}{l}\text { Erwartete } \\
\text { Karrierehindernisse }\end{array}$ & 3,22 & 3,00 & $\begin{array}{l}\text { Beurteilung von } 6 \text { Aussagen auf einer 5er- } \\
\text { Likert-Skala von } 1 \text { für "Trifft gar nicht zu" } \\
\text { bis } 5 \text { "Trifft sehr gut zu", dann Dimensi- } \\
\text { onsreduktion durch Faktorenanalyse }\end{array}$ \\
\hline Extraversion & 4,69 & 5,00 & \multirow{5}{*}{$\begin{array}{l}\text { Beurteilung von jeweils } 3 \text { Aussagen pro } \\
\text { Faktor auf einer 7er-Likert-Skala von } 1 \\
\text { für "Trifft gar nicht zu" bis } 7 \text { "Trifft sehr } \\
\text { gut zu", dann Dimensionsreduzierung } \\
\text { durch Faktorenanalyse }\end{array}$} \\
\hline Gewissenhaftigkeit & 5,66 & 6,00 & \\
\hline Verträglichkeit & 5,34 & 5,00 & \\
\hline Neurotizismus & 3,49 & 3,00 & \\
\hline Offenheit für Erfahrung & 4,73 & 5,00 & \\
\hline
\end{tabular}

Legende: $\mathrm{P}=$ Partnerin.

Quelle: Eigene Erstellung

\subsection{Ergebnisse}

Um zunächst die in Abschnitt 3 formulierten Hypothesen zu prüfen, werden drei vollständige Schätzmodelle mit den abhängigen Variablen „Inanspruchnahme von Elternzeit: ja (1) - nein 
$(0)$ “, „Dauer der Inanspruchnahme > 2 Monate: ja (1) - nein (0)“ und „Dauer der Inanspruchnahme (in Monaten)" gerechnet (siehe Tabelle 2). Für die ersten beiden Schätzungen (Hypothesen 1 und 2) werden aufgrund des binären Charakters der abhängigen Variable logistische Regressionen durchgeführt (Modelle 1 und 2), für die dritte Schätzung (Hypothese 3) eine einfache OLS-Regression (Modell 3). Die für Modell 1 und 2 abgedruckten Effektkoeffizienten geben den Einfluss der abhängigen Variable auf das sogenannte Chancenverhältnis an, d.h. das Verhältnis zwischen der Wahrscheinlichkeit einer Inanspruchnahme von Elternzeit und der Wahrscheinlichkeit, Elternzeit nicht in Anspruch zu nehmen. Effektkoeffizienten größer 1 bilden entsprechend einen positiven Zusammenhang zwischen der erklärenden Variable und der zu erklärenden Variable ab, Effektkoeffizienten kleiner 1 einen negativen Zusammenhang.

Tabelle 2: (Dauer der) Inanspruchnahme von Elternzeit

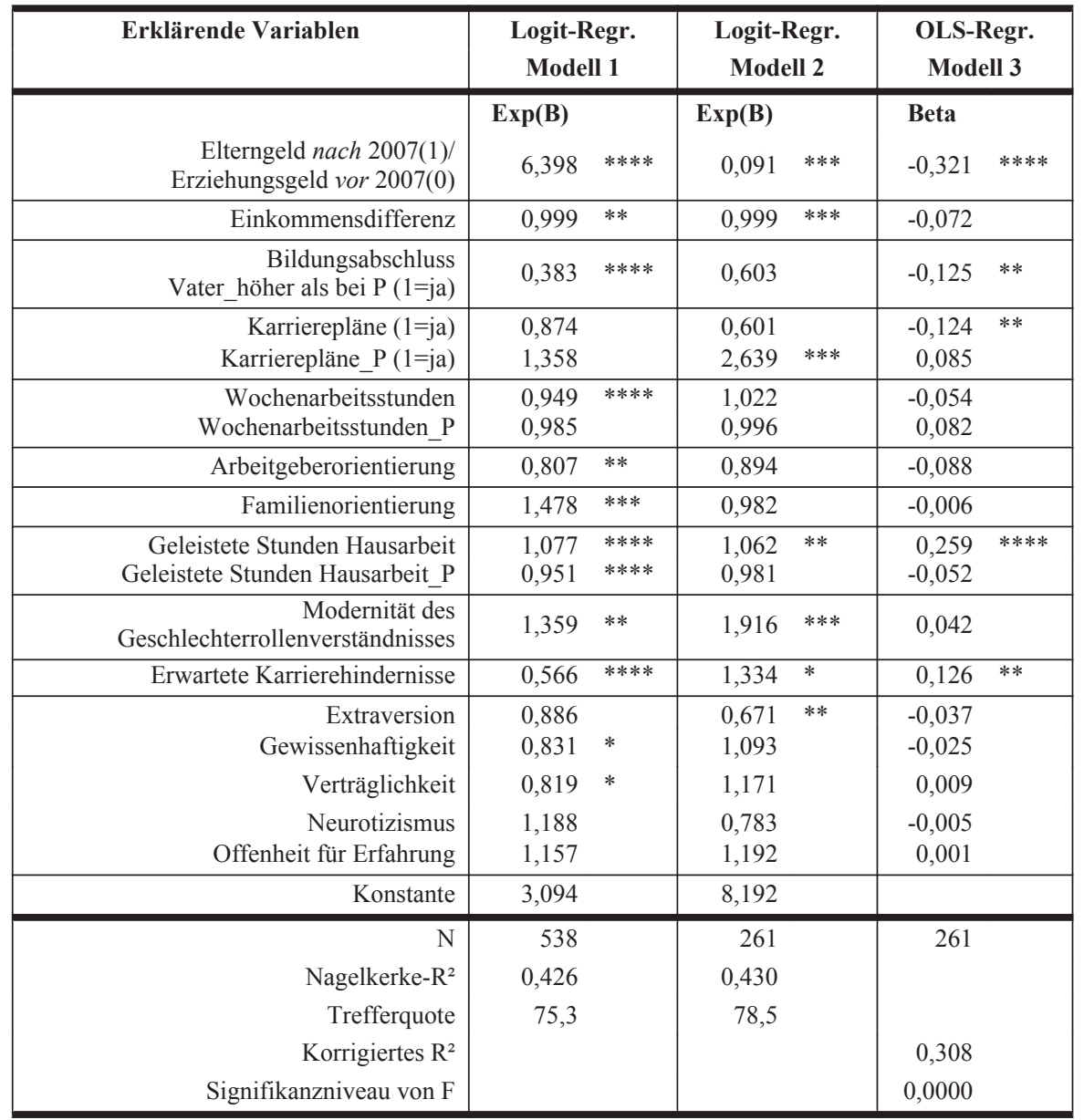

Signifikanzniveaus: $* * * * \leq 0,001 ; * * * \leq 0,01 ; * * \leq 0,05 ; * \leq 0,1$.

Legende: $\mathrm{P}=$ Partnerin.

Quelle: Eigene Berechnungen. 
Als Ergebnis lässt sich zunächst mit Bezug auf die formulierten Hypothesen feststellen, dass die Wahrscheinlichkeit, dass Väter Elternzeit beanspruchen (Modell 1), mit der Elterngeldreform deutlich angestiegen ist. Der Zusammenhang ist nicht nur statistisch höchst signifikant, sondern auch von der Größenordnung her bedeutsam: Das Chancenverhältnis einer väterlichen Inanspruchnahme von Elternzeit hat sich nach der Reform um einen Faktor 6 erhöht. Das heißt, Hypothese 1 wird durch unsere Daten unterstützt. Des Weiteren ist zu erkennen, dass die Wahrscheinlichkeit, dass Väter, die Elternzeit in Anspruch nehmen, dies für einen Zeitraum von über zwei Monaten tun, nach der Elterngeldreform statistisch höchst signifikant gesunken ist (Modell 2). Entsprechend hat sich die durchschnittliche Dauer der Inanspruchnahme von Elternzeit durch Väter, die in Elternzeit gehen, seit der Elterngeldreform statistisch höchst signifikant verkürzt (Modell 3). Dieser Effekt könnte dabei allerdings auch dadurch zustande kommen, dass die maximale Bezugsdauer von Eltergeld seit der Elterngeldreform nur noch 14 Monate statt vormals 24 Monate beträgt (wobei eine 24-monatige Inanspruchnahme des vollen Erziehungsgelds auch vor der Reform nur für eine Teilpopulation möglich war). Von den 67 Vätern in unserem Datensatz, die vor dem 1.1.2007 Elternzeit in Anspruch nahmen und Auskunft über die Dauer des Bezugs von Elterngeld (bzw. genauer: Erziehungsgeld) machten, gibt ca. ein Drittel eine Bezugsdauer von über 14 Monaten an. Insgesamt werden damit sowohl Hypothese 2 als auch Hypothese 3 ebenfalls gestützt. Mit anderen Worten, die Elterngeldreform hat dazu geführt, dass Väter zwar eher in Elternzeit gehen, aber dafür im Durchschnitt kürzer, da im Wesentlichen die Vätermonate in Anspruch genommen werden bzw. Bezugsdauern von über 14 Monaten nicht mehr möglich sind. Ob damit die erhoffte „Väterrevolution“ ins Rollen gebracht werden konnte, sei dahingestellt. So weisen Kritiker des Elterngelds darauf hin, dass insbesondere gut verdienende Elternteile (in der Regel die Väter) ihre Erwerbstätigkeit nur unterbrechen, um das an die Vätermonate geknüpfte Elterngeld ausschöpfen zu können, dass aber die eigentlich vom BMFSFJ angestrebte Anreizsetzung für Väter, sich vermehrt und über einen längeren Zeitraum aktiv an Elternzeit und Kindererziehung zu beteiligen, ausbleibt. Darüber hinaus sind viele Eltern mit der Einführung des Elterngelds sogar schlechter gestellt als zuvor: So stand beispielsweise vielen arbeitslosen oder studierenden Eltern bzw. Eltern in Ausbildung, Hausfrauen und -männern vor der Einführung des Elterngelds Erziehungsgeld in Höhe von 300 Euro für 24 Monate zu. Seit dem 1.1.2007 erhalten sie Elterngeld in gleicher Höhe für nur noch maximal 14 Monate. Im Gegensatz zu den Akademikern und Gutverdienern, die am stärksten von der Einführung des Elterngelds profitieren, fühlen sie sich als die Verlierer des Elterngelds.

Bezüglich der weiteren potenziellen Einflussfaktoren auf die väterliche Entscheidung für oder wider Elternzeit (Modell 1) zeigen sich keine kontra-intuitiven Ergebnisse. Allerdings ist nur ein Teil der Zusammenhänge statistisch signifikant. Bezogen auf das berufliche Umfeld zeigt sich, dass die Wahrscheinlichkeit einer väterlichen Inanspruchnahme von Elternzeit (umso) geringer ist, (a) je mehr der Vater im Vergleich zu seiner Partnerin verdient, (b) wenn der Vater einen höheren Bildungsabschluss hat als seine Partnerin, (c) je mehr Arbeitsstunden er leistet und (d) je ausgeprägter seine Arbeitgeberorientierung ist. Bezogen auf das familiäre Umfeld ergibt sich, dass die Wahrscheinlichkeit einer väterlichen Inanspruchnahme von Elternzeit umso höher ist, je ausgeprägter seine Familienorientierung ist und je mehr Stunden Hausarbeit er bereits vor der Entscheidung über Elternzeit leistete. Hinsichtlich des gesellschaftlichen-sozialen Umfelds zeigt sich, dass die Wahrscheinlichkeit einer väterlichen Inanspruchnahme von Elternzeit umso höher ist, je moderner das Geschlechterrollenverständnis des Vaters und je geringer die erwarteten resp. befürchteten Karrierehindernisse. Von den Persönlichkeitsfaktoren lässt sich lediglich für „Gewissenhaftigkeit“" und „Verträglichkeit“" ein statistisch signifikanter Zusammenhang zur Wahrscheinlichkeit einer Inanspruchnahme durch den Vater nachweisen. Beides, eine ausgeprägte Gewissenhaftigkeit wie auch eine ausgeprägte Verträglichkeit, reduzieren die Wahrscheinlichkeit einer Inanspruchnahme von Elternzeit durch den Vater. 
Auch bei der Entscheidung für oder wider die Inanspruchnahme von Elternzeit über einen Zeitraum von mehr als zwei Monaten (Modell 2) gibt es keine überraschenden Befunde. Aus dem beruflichen Umfeld sind die Einkommensdifferenz zur Partnerin sowie die Karrierepläne der Partnerin statistisch signifikant mit der Entscheidung des Vaters für oder wider eine Inanspruchnahme von Elternzeit mit einer Dauer von über zwei Monaten korreliert: Je mehr der Vater im Vergleich zu seiner Partnerin verdient, umso eher schreckt er vor einer Inanspruchnahme von Elternzeit zurück. Stehen bei seiner Partnerin allerdings unmittelbar Karriereschritte bevor, dann steigt die Wahrscheinlichkeit einer väterlichen Inanspruchnahme von Elternzeit. Im familiären Umfeld spielt eine Rolle, in welchem Umfang sich der Vater bereits vor der Entscheidung über Elternzeit in der Hausarbeit engagierte. Je mehr dies der Fall war, umso eher entscheidet er sich, mehr als nur zwei Monate Elternzeit in Anspruch zu nehmen. Im gesellschaftlich-sozialen Umfeld zeigt sich einerseits analog zum Zusammenhang bei der Frage nach der Inanspruchnahme von Elternzeit, dass die Wahrscheinlichkeit, dass der Vater sich zu einer Inanspruchnahme von Elternzeit im Umfang von mehr als zwei Monaten entschließt, mit zunehmender Modernität seines Geschlechterrollenverständnisses ansteigt. Überraschenderweise sind höhere erwartete Karrierehindernisse jedoch mit einer höheren Wahrscheinlichkeit, dass der Vater sich zu einer Inanspruchnahme von Elternzeit im Umfang von mehr als zwei Monaten entschließt, verbunden. Dieser Befund ist dabei jedoch nicht zwingend dahingehend zu interpretieren, dass höhere erwartete Karrierehindernisse zu einer längeren Inanspruchnahme von Elternzeit führen, sondern könnte auch dadurch zustande gekommen sein, dass die befragten Väter in der Regel erst ex post, d.h. nachdem sie Elternzeit in Anspruch genommen hatten, ihre ex ante-Erwartungen über die mutmaßlichen Karriere-Effekte einer Inanspruchnahme von Elternzeit formuliert haben. Somit könnten sich in der Folge ex anteErwartungen und ex post-Erfahrungen auf eine Art und Weise miteinander vermischt haben, dass sie für die Befragten nicht mehr einzeln identifizierbar waren. Es besteht damit die Möglichkeit, dass insbesondere Väter, die sich für eine lange Dauer der Elternzeit-Inanspruchnahme entschieden haben und in der Folge (möglicherweise auch unerwartet) Karrierehindernisse erfahren mussten, systematisch, aber verzerrt durch ihre ex post-Erfahrungen, höhere Zustimmungsgrade zu den Karrierehindernis-Items äußerten. Entsprechend ist die Variable ,,erwartete Karrierehindernisse" mit einer gewissen Vorsicht zu betrachten. Was die Persönlichkeitsfaktoren anbelangt, so ist eine ausgeprägte Extraversion offenbar hinderlich für die Inanspruchnahme von Elternzeit im Umfang von über zwei Monaten.

Bezüglich der Dauer der Inanspruchnahme von Elternzeit (Modell 3) sind nur wenige der potenziell erklärenden Variablen signifikant: Die Dauer der Inanspruchnahme von Elternzeit ist (umso) höher, wenn der Vater einen geringeren Bildungsabschluss als die Partnerin hat, wenn für ihn selbst keine Karriereschritte unmittelbar bevorstehen und je mehr Zeit er bereits vor der Entscheidung über Elternzeit in Hausarbeit gesteckt hat. Für die Interpretation des ebenfalls als positiv gemessenen Zusammenhangs zwischen erwarteten Karrierehindernissen und Dauer der Inanspruchnahme von Elternzeit gilt dabei das bereits im Zusammenhang mit der abhängigen Variable „Dauer der Inanspruchnahme > 2 Monate: ja (1) - nein (0)“ Gesagte.

In einem nächsten Schritt betrachten wir, ob und inwiefern sich die Determinanten der väterlichen Entscheidung für oder wider Elternzeit durch und mit der Reform verändert haben (siehe Tabelle 3). Hierzu führen wir zwei Modell-Schätzungen mit der abhängigen Variable „Inanspruchnahme von Elternzeit: ja (1) - nein (0)“ für unterschiedliche Teilpopulationen an Vätern durch. Während Modell 4 auf der Teilpopulation der Väter basiert, die sich für ein vor dem 1.1.2007 geborenes Kind für oder wider die Inanspruchnahme von Elternzeit entschieden haben, liegt Modell 5 die Teilpopulation der Väter zugrunde, die die Entscheidung für ein nach dem 1.1.2007 geborenes Kind zu treffen hatten. Während es in den Modellen 1-3 um die Überprüfung konkreter Hypothesen zum direkten Einfluss der Elterngeldreform auf 
die Inanspruchnahme von Elternzeit durch Väter ging, handelt es sich an dieser Stelle des Beitrags um eine explorative Analyse der indirekten Reformeffekte.

Tabelle 3: Inanspruchnahme von Elternzeit vor versus nach 2007

\begin{tabular}{|c|c|c|c|c|}
\hline Erklärende Variablen & \multicolumn{2}{|c|}{$\begin{array}{c}\text { Logit-Regr. } \\
\text { Modell 4 - vor } 2007\end{array}$} & \multicolumn{2}{|c|}{$\begin{array}{c}\text { Logit-Regr. } \\
\text { Modell } 5 \text { - nach } 2007\end{array}$} \\
\hline Einkommensdifferenz & $\begin{array}{r}\operatorname{Exp}(\mathbf{B}) \\
0,999\end{array}$ & $* *$ & $\begin{array}{r}\operatorname{Exp}(\mathbf{B}) \\
0,999\end{array}$ & \\
\hline $\begin{array}{r}\text { Bildungsabschluss Vater_höher als bei P } \\
\qquad(1=\mathrm{ja})\end{array}$ & 0,113 & $* * * *$ & 0,428 & $* * *$ \\
\hline $\begin{array}{r}\text { Karrierepläne }(1=\mathrm{ja}) \\
\text { Karrierepläne_P }(1=\mathrm{ja})\end{array}$ & $\begin{array}{l}1,159 \\
2,227\end{array}$ & $*$ & $\begin{array}{l}0,858 \\
0,911\end{array}$ & \\
\hline $\begin{array}{r}\text { Wochenarbeitsstunden } \\
\text { Wochenarbeitsstunden_P }\end{array}$ & $\begin{array}{l}0,974 \\
0,983\end{array}$ & & $\begin{array}{l}0,939 \\
0,987\end{array}$ & $* * * *$ \\
\hline Arbeitgeberorientierung & 1,090 & & 0,756 & $* *$ \\
\hline Familienorientierung & 1,047 & & 1,549 & $* * *$ \\
\hline $\begin{array}{r}\text { Geleistete Stunden Hausarbeit } \\
\text { Geleistete Stunden Hausarbeit_P }\end{array}$ & $\begin{array}{l}1,064 \\
0,914\end{array}$ & $\begin{array}{l}* * \\
* * * *\end{array}$ & $\begin{array}{l}1,093 \\
0,951\end{array}$ & $\begin{array}{l}* * * * \\
* * * *\end{array}$ \\
\hline $\begin{array}{r}\text { Modernität } \\
\text { Geschlechterrolleneinstellung }\end{array}$ & 1,550 & $*$ & 1,254 & $*$ \\
\hline Erwartete Karrierehindernisse & 0,458 & $* * *$ & 0,580 & $* * * *$ \\
\hline $\begin{array}{r}\text { Extraversion } \\
\text { Gewissenhaftigkeit }\end{array}$ & $\begin{array}{l}0,483 \\
1,075\end{array}$ & $* * * *$ & $\begin{array}{l}1,039 \\
0,870\end{array}$ & \\
\hline Verträglichkeit & 0,928 & & 0,747 & $* *$ \\
\hline $\begin{array}{r}\text { Neurotizismus } \\
\text { Offenheit für Erfahrung }\end{array}$ & $\begin{array}{l}1,287 \\
0,899\end{array}$ & & $\begin{array}{l}1,130 \\
1,170\end{array}$ & \\
\hline Konstante & 4,447 & & 30,722 & $* * *$ \\
\hline $\mathrm{N}$ & 200 & & 374 & \\
\hline Nagelkerke- ${ }^{2}$ & 0,486 & & 0,325 & \\
\hline Trefferquote & 81,0 & & 73,5 & \\
\hline
\end{tabular}

Signifikanzniveaus: $* * * * \leq 0,001 ; * * * \leq 0,01 ; * * \leq 0,05 ; * \leq 0,1$.

Legende: $\mathrm{P}=$ Partnerin.

Quelle: Eigene Berechnungen.

Vergleicht man Modell 4 und 5, so zeigt sich eine Reihe von Unterschieden, was die Wirksamkeit einzelner Determinanten der Inanspruchnahme von Elternzeit durch Väter vor und nach der Elterngeldreform anbelangt. Zu den Variablen, die vor der Elterngeldreform noch in einem statistisch signifikanten Zusammenhang mit der Inanspruchnahme von Elternzeit durch Väter standen, aber seit dem nicht mehr, gehören die Differenz zwischen dem Einkommen des Vaters und dem seiner Partnerin, die Frage, ob die Partnerin in nächster Zukunft konkrete Karriereschritte plant und der Grad der Extraversion des Vaters. Seit Inkrafttreten der Elterngeldreform führen ein höheres Einkommen des Vaters im Vergleich zu dem der Partnerin, eine Verneinung der Frage, ob die Partnerin konkrete Karriereschritte plant, sowie ein höheres Maß an väterlicher Extraversion nicht mehr dazu, dass die Wahrscheinlichkeit für die väterliche Inanspruchnahme von Elternzeit sinkt. Die Inanspruchnahme von Elternzeit durch Väter ist vielmehr statistisch unabhängig davon, ob der Vater im Vergleich zu seiner Partnerin gut oder schlecht verdient, ob die Partnerin konkrete Karriereschritte plant oder nicht und zu welchem 
Grad der Vater extrovertiert ist. Offenbar nehmen im Vergleich zu ihrer Partnerin gut verdienende Väter Elternzeit nunmehr genauso wahr wie die im Vergleich zu ihrer Partnerin weniger verdienenden Väter. Gleiches gilt für Väter, deren Partnerinnen keine Karriereschritte planen, im Vergleich zu solchen, für deren Partnerinnen konkrete Karriereschritte anstehen, sowie auch für extrovertierte im Vergleich zu introvertierten Vätern. Hier hat die Reform offenbar durchaus etwas bewegt: Weder ,braucht“ es ein geringeres Einkommen des Vaters im Vergleich zur Partnerin noch anstehende konkrete Karriereschritte der Partnerin noch ein besonders hohes $\mathrm{Maß}$ an Extraversion, damit Väter das Wagnis Elternzeit eingehen. Stattdessen spielen nunmehr verstärkt die Arbeitgeberorientierung (negativ korreliert mit der väterlichen Inanspruchnahme von Elternzeit) und die Familienorientierung (positiv korreliert mit der väterlichen Inanspruchnahme von Elternzeit) eine Rolle sowie die berufliche Belastung und die Verträglichkeit des Vaters (jeweils negativ korreliert mit der väterlichen Inanspruchnahme von Elternzeit). Allerdings ist im Hinblick auf eine Interpretation dieser Ergebnisse zu beachten, dass mit der größeren Fallzahl in Modell 5 auch die Wahrscheinlichkeit steigt, statistisch signifikante Zusammenhänge ermitteln zu können. In beiden Modellen, d.h. sowohl vor 2007 als auch seit 2007 statistisch signifikant mit der väterlichen Inanspruchnahme von Elternzeit verknüpft sind die Fragen, (a) ob Vater oder Mutter über einen höheren Bildungsabschluss verfügen, (b) nach den durch den Vater und (c) dessen Partnerin bereits vor der väterlichen Inanspruchnahme von Elternzeit geleisteten Stunden an Hausarbeit, (d) die Modernität des väterlichen Geschlechterrollenverständnisses und (e) der Grad, zu dem Karrierehindernisse bei Inanspruchnahme von Elternzeit durch Väter befürchtet werden. D.h. damals wie heute gilt, dass eine höhere vorherige Beteiligung des Vaters an der Hausarbeit sowie auch ein moderneres Geschlechterrollenverständnis des Vaters die Wahrscheinlichkeit einer väterlichen Inanspruchnahme von Elternzeit erhöhen, während ein höherer Bildungsabschluss des Vaters im Vergleich zu dem der Partnerin sowie die Befürchtung des Vaters, infolge einer Inanspruchnahme von Elternzeit Karrierehindernisse erfahren zu müssen, die Wahrscheinlichkeit einer väterlichen Inanspruchnahme von Elternzeit reduzieren.

Wenn und insofern es durch die Elterngeldreform gelingt, tragfähige Impulse in Richtung eines moderneren Geschlechterrollenverständnis zu induzieren (in Bezug auf die Einstellungen der Väter, aber auch in Bezug auf das ,gelebte Geschlechterrollenverständnis“, etwa wenn es um die Verteilung der Hausarbeit zwischen den Geschlechtern geht) und/oder die soziale Akzeptanz für eine väterliche Inanspruchnahme von Elternzeit zu erhöhen (geringere erwartete Karrierehindernisse), dann dürften sich die Effekte der Elterngeldreform noch deutlich verstärken - und möglicherweise irgendwann zu messbaren Auswirkungen jenseits der Inanspruchnahme der Vätermonate führen. Unsere Daten liefern dabei bereits erste Ansatzpunkte für entsprechende Effekte: Zwar lassen sich im Rahmen eines ergänzend durchgeführten tTests auf Mittelwertdifferenzen weder bei der Modernität des Geschlechterrollenverständnisses noch bei der gelebten Arbeitsteilung im Haushalt signifikante Unterschiede zwischen den Teilpopulationen der befragten Väter, die vor dem 1.1.2007 Elternzeit in Anspruch genommen haben, und solchen, die dies seit dem 1.1.2007 getan haben, feststellen, allerdings besteht ein statistisch höchst signifikanter Zusammenhang zwischen der Einschätzung der erwarteten Karrierehindernisse und dem Zeitpunkt der Elternzeit-Inanspruchnahme (vor bzw. nach 2007): Seit der Elterngeldreform erwarten Väter im Falle einer Inanspruchnahme von Elternzeit in einem signifikant geringeren Ausmaß Karrierehindernisse als zuvor. Also doch: der Beginn einer „Väterrevolution?“

\section{Zusammenfassung}

Seit der Einführung des Elterngeldes zum 01. Januar 2007 ist der Anteil der Väter in Elternzeit über das gesamte Jahr 2007 hinweg zwar deutlich angestiegen; dennoch stellen Väter in Elternzeit nach wie vor eine Minderheit dar. An diesem Punkt setzt der vorliegende Beitrag an 
und untersucht, wie die Elterngeldreform 2007 die Entscheidung eines berufstätigen Vaters für oder wider die Inanspruchnahme von Elternzeit beeinflusst hat. Als Ergebnis der empirischen Analyse, in deren Verlauf 1.290 Online-Fragebögen berufstätiger Väter ausgewertet werden konnten, zeigt sich, dass die Elterngeldreform die Wahrscheinlichkeit der väterlichen Inanspruchnahme von Elternzeit zwar signifikant erhöht hat, dass Väter seit Inkrafttreten der Reform aber im Durchschnitt kürzer in Elternzeit gehen und im Wesentlichen die so genannten Vätermonate in Anspruch nehmen. Dies gilt auch nach Kontrolle einer Vielzahl potenzieller Determinanten der väterlichen Inanspruchnahme von Elternzeit aus dem beruflichen, familiären und gesellschaftlich-sozialen Umfeld sowie nach Kontrolle von Persönlichkeitsfaktoren. Was die übrigen Einflussfaktoren der väterlichen Inanspruchnahme von Elternzeit anbelangt, so gibt unsere Analyse erste tentative Hinweise darauf, dass die Elterngeldreform auch in dem Sinne zu einer Veränderung des väterlichen Entscheidungskalküls für oder wider die Inanspruchnahme von Elternzeit geführt hat, als dass auf der einen Seite vormals wirksame Determinanten des Kalküls in ihrer Wirkung abgeschwächt wurden und auf der anderen Seite vormals weniger bedeutsame Determinanten in ihrer Wirksamkeit gestärkt wurden. Nach wie vor sind Faktoren aus dem beruflichen, familiären und gesellschaftlich-sozialen Umfeld sowie vereinzelt auch Persönlichkeitsfaktoren relevant für die väterliche Entscheidung für oder wider Elternzeit. Je mehr es im Zuge der Elterngeldreform gelingt, diese übrigen Entscheidungsfaktoren zu beeinflussen, umso eher wird es in Zukunft Wirkungen der Reform geben, die über die reine Inanspruchnahme der Vätermonate hinausgehen.

\section{Literatur}

Behnke, Cornelia / Michael Meuser (2003): Vereinbarkeitsmanagement, Die Herstellung von Gemeinschaft bei Doppelkarrieren, in: Soziale Welt 54, S. 163-174.

Benard, Cheryl / Edit Schlaffer (1991): Sagt uns, wo die Väter sind. Von Arbeitssucht und Fahnenflucht des zweiten Elternteils. Reinbek.

Bianchi, Suzanne M. / Melissa A. Milkie / Liana C. Sayer / John P. Robinson (2000): Is anyone doing the housework? Trends in the gender division of household labor, in: Social Forces 79 / 1, S. 191-234.

Blankenhorn, David (1995): Fatherless America: Confronting our most urgent social problem, New York.

Boeykens, Lily (1993): The father in focus, in: Materialien zur Bevölkerungswissenschaft, Sonderheft 21, Wiesbaden.

Bulanda, Roland E. (2004): Paternal involvement with children: The influence of gender ideologies, in: Journal of Marriage and Family 66 / 1, S. 40-45.

Bundesministerium für Familie, Senioren, Frauen, Jugend (2004): Bericht über die Auswirkungen der $\S \S 15$ und 16 Bundeserziehungsgeldgesetz.

Bundesminsterium für Familie, Senioren, Frauen, Jugend (2007): Elterngeld und Elternzeit. Das Bundeselterngeld- und Elternzeitgesetz, abgerufen am 24.9.2007 unter http://www.bmfsfj.de/ bmfsfj/generator/Kategorien/Publikationen/publikationsliste, did=89272.html.

Bundesminsterium für Familie, Senioren, Frauen, Jugend (2008 a): Erfolgsbilanz nach einem Jahr: Das Elterngeld wirkt. Pressebericht vom 29.2.2008, abgerufen am 29.2.2008 unter. http://www.bmfsfj.de/ bmfsfj/generator/Politikbereiche/familie,did=108302.html.

Bundesminsterium für Familie, Senioren, Frauen, Jugend (2008 b): Früher beruflicher Wiedereinstieg von Eltern. Ein Gewinn für Unternehmen und ihre Beschäftigten, Berlin.

Coltrane, Scott (1996): Family man: Fatherhood, housework, and gender equity, New York.

Coverman, Shelley (1985): Explaining husbands' participation in domestic labor, in: The Sociological Quarterly 26, S. 81-97. 
Döge, Peter / Rainer Volz (2002): Wollen Frauen den neuen Mann? - Traditionelle Geschlechterbilder als Blockaden von Geschlechterpolitik, in: Konrad-Adenauer-Stiftung e.V. (Hrsg.): Zukunftsforum Politik, St. Augustin.

Engelhardt, Henriette / Ben Jan (2004): Halbe Kraft voraus? Arbeitseinsatz, berufliche Segregation und Löhne von Frauen auf dem Schweizer Arbeitsmarkt, in: Soziale Welt 55, S. 205-224.

Fthenakis, Wassilios E. (1985): Väter I/II, Zur Psychologie der Vater-Kind-Beziehung. Weinheim.

Gerlitz, Jean- Yves / Jürgen Schupp (2005): Zur Erhebung der Big Five-basierten Persönlichkeitsmerkmale im SOEP, Dokumentation der Instrumententwicklung BFI-S auf Basis des SOEP-Pretests 2005, Research Notes 4, Deutsches Institut für Wirtschaftsforschung.

Gornick, Janet C. / Marcia K. Meyers (2008): Creating gender egalitarian societies: an agenda for reform, in: Politics and Society 36, S. 313-349.

Griswold, Robert L. (1993): Fatherhood in America. A History, New York.

Hill, Paul B. / Johannes Kopp (1995): Familiensoziologie: Grundlagen und theoretische Perspektiven, Stuttgart.

Hofferth, Sandra (2003): Race / ethnic differences in father involvement in two-parent families, in: Journal of Family Issues 24, S. 185-216.

Höpflinger, François / Maria Charles (1990): Innerfamiliale Arbeitsteilung: Mikro-soziologische Erklärungsansätze und empirische Beobachtungen, in: Zeitschrift für Familienforschung 2, S. 87-113.

Kirchmeyer, Catherine (2002): Gender differences in managerial careers: Yesterday, today and tomorrow, in: Journal of Business Ethics 37, S. 5-24.

Kreyenfeld, Michaela / Esther Geisler (2009): Against all Odds: Fathers' Use of Parental Leave in Germany, Rostock.

Künzler, Jan (1994): Familiale Arbeitsteilung, Die Beteiligung der Männer an der Hausarbeit, in: Institut Frau und Gesellschaft (Hrsg.): Reihe Theorie und Praxis der Frauenforschung, Bielefeld.

Lappegård, Trude (2008): Changing the gender balance in caring: fatherhood and the division of parental leave in Norway, in: Population Research and Policy Review 27, S. 139-159.

Merz, Monika (2004): Women's Hours of Market Work in Germany: The Role of Parental Leave, Bonn.

Metz-Göckel, Sigrid (1988): Väter und Väterlichkeit, Zur alltäglichen Beteiligung der Väter an der Erziehungsarbeit, in: Zeitschrift für Sozialisationsforschung und Erziehungssoziologie 8, S. 264-280.

Oberndorfer, Rotraut / Harald Rost (2002): Auf der Suche nach den neuen Vätern, Familien mit nichttraditioneller Verteilung von Erwerbs- und Familienarbeit, in: Staatsinstitut für Familienforschung an der Universität Bamberg (ifb), Forschungsbericht Nr. 5.

Oberndorfer, Rotraut (1993): Aufgabenteilung in Partnerschaften, in: Nauck, Bernhard (Hrsg.): Lebensgestaltung von Frauen, Eine Regionalanalyse zur Integration von Familien- und Erwerbstätigkeit im Lebensverlauf, Weinheim / München.

Ondrich, Jan / C. Katharina Spieß / Qing Yang (1996): Barefoot and in a German Kitchen: Federal Parental Leave and Benefit Policy and the Return to Work after Childbirth in Germany', in: Journal of Population Economics 9/3, S. 247-266.

Palkovitz, Rob / Loren Marks (2002): Die Kultivierung von Vaterschaft und Mutterschaft: Eine Analyse von Trends in der Familienerziehung, in: Wassilios E. Fthenakis / Martin R. Textor, (Hrsg.): Mutterschaft, Vaterschaft, Weinheim, S. 120-128.

Peinelt-Jordan, Klaus (1996): Männer zwischen Familie und Beruf, Ein Anwendungsfall für die Individualisierung der Personalpolitik, München / Mering.

Pleck, Joseph H. (1997): Paternal involvement: Levels, sources, and consequences, in: Lamb, Michael E. (Hrsg.): The role of the father in child development. 3. Auflage, New York, S. 66-103.

Pylkkänen, Elina / Nina Smith (2003): Career Interruptions due to Parental Leave - A Comparative Study of Denmark and Sweden, in: OECD Social, Employment and Migration Working Papers: Directorate 
for Employment, Labour and Social Affairs (DELSA) / Employment, Labour and Social Affairs Committee (ELSA) / OECD Social Employment and Migration, Arbeitspapier Nr. 1, Paris.

Sayer, Liana C. / Suzanne M. Bianchi / John P. Robinson (2004): Are parents investing less in children? Trends in mothers' and fathers' time with children, in: American Journal of Sociology 110, S. 1-43.

Schumann, Siegfried (2005): Persönlichkeit. Eine vergessene Größe der empirischen Sozialforschung, Wiesbaden.

Spieß, C. Katharina (2006): Elterngeld: Kürzere Erwerbspausen von Müttern erwartet. In: DIW Wochenbericht 48, S. 689-693.

Spieß, C. Katharina / Katharina Wrohlich (2008): The Parental Leave Benefit Reform in Germany: Costs and Labour Market Outcomes of Moving towards the Nordic Model, in: Population Research and Policy Review 5, S. 575-591.

Sundström, Marianne / Ann-Zofie Duvander (2002): Gender division of childcare and the sharing of parental leave among new parents in Sweden, in: European Sociological Review 18, S. 433-447.

Vaskovics, Laszlo / Harald Rost (1999): Väter und Erziehungsurlaub, in: Schriftenreihe des Bundesministeriums für Familie, Senioren, Frauen und Jugend, Band 179, Stuttgart.

Vaskovics, Laszlo / Marina Rupp (1995): Partnerschaftskarrieren. Entwicklungspfade nichtehelicher Lebensgemeinschaften, Opladen.

Yeung, W. Jean / John F. Sandberg / Pamela E. Davis-Kean / Sandra L. Hofferth (2001): Children's Time with Fathers in Intact Families, in: Journal of Marriage and the Family 63 / 1, S. 136-154.

Prof. Dr. Kerstin Pull

Universität Tübingen

Lehrstuhl für Personal und Organisation

Nauklerstr. 47

72074 Tübingen

Kerstin.Pull@uni-tuebingen.de 